\title{
Bioinformatic analysis of computational identified differentially expressed genes in tumor stoma of pregnancy-associated breast cancer
}

\author{
QIAN ZHOU ${ }^{1 *}$, ERHU SUN $^{1 *}$, LIJUN LING $^{2}$, XIAOFENG LIU ${ }^{1}$, MIN ZHANG ${ }^{1}$, HONG YIN $^{1}$ and CHENG LU ${ }^{1}$ \\ ${ }^{1}$ Department of Breast, Nanjing Maternity and Child Health Care Hospital, Obstetrics and Gynecology Hospital \\ Affiliated to Nanjing Medical University, Nanjing, Jiangsu 210004; ${ }^{2}$ Department of Breast Surgery, \\ The First Affiliated Hospital of Nanjing Medical University, Nanjing, Jiangsu 210029, P.R. China
}

Received January 12, 2016; Accepted February 9, 2017

DOI: $10.3892 / \mathrm{mmr} .2017 .6947$

\begin{abstract}
The present study aimed to screen the differentially expressed genes (DEGs) in tumor-associated stroma of pregnancy-associated breast cancer (PABC). By analyzing Affymetrix microarray data (GSE31192) from the Gene Expression Omnibus database, DEGs between tumor associated stromal cells and normal stromal cells in PABC were identified. Gene Ontology (GO) function and pathway enrichment analyses for the DEGs were then performed, followed by construction of a protein-protein interaction (PPI) network. A total of 94 upregulated and 386 downregulated DEGs were identified between tumor associated stromal cells and normal stromal cells in patients with PABC. The upregulated DEGs were primarily enriched in the cytokine-cytokine receptor interaction pathway and GO terms associated with the immune response, which included the DEGs of interleukin 18 (IL18) and cluster of differentiation 274 (CD274). The downregulated DEGs were primarily involved in GO terms associated with cell surface receptor linked signal transduction and pathways of focal adhesion and pathways in cancer. In the PPI network, nodes of jun proto-oncogene (JUN), FBJ murine osteosarcoma viral oncogene homolog (FOS), V-myc avian myelocytomatosis viral oncogene homolog $(M Y C)$, and alpha-smooth muscle actin (ACTA2) had higher degrees. The hub genes of JUN, FOS, MYC and ACTA2, as well as the
\end{abstract}

Correspondence to: Dr Hong Yin or Dr Cheng Lu, Department of Breast, Nanjing Maternity and Child Health Care Hospital, Obstetrics and Gynecology Hospital Affiliated to Nanjing Medical University, 123 Mochou Road, Tianfeixiang, Nanjing, Jiangsu 210004, P.R. China

E-mail: yinhhshsh@hotmail.com

E-mail: chengludhh@163.com

*Contributed equally

Key words: pregnancy-associated breast cancer, differentially expressed gene, enrichment analysis, protein-protein interaction network
DEGs IL18 and CD274 that were associated with the immune response in GO terms may exert important functions in the molecular mechanisms of PABC. These genes may be used as new molecular targets in the treatment of this disease.

\section{Introduction}

Pregnancy-associated breast cancer (PABC) is a primary breast cancer characterized by breast lumps, breast thickening and changes in breast shape or pits of skin (1). It occurs during pregnancy or within one year postpartum, with an increased risk of metastasis and mortality. The incidence rate of PABC is $\sim 0.76-3.80 \%$ and the age is $23-47$ years old (2). The incidence of PABC has risen due to women delaying childbearing (3). Despite efforts being made to improve the diagnosis and treatment of PABC, the development of clinically validated detection markers represents a great challenge. Therefore, it is important to understand the underlying molecular mechanisms of PABC to provide the treatment basis for patients with PABC.

Multiple epidemiological studies have reported that the genetic factors, including breast cancer 1, early onset (BRCAl) or BRCA2 mutations, are involved in increasing the incidence of PABC (4-6). Overexpression of tumor protein p53 and Erb-B2 receptor tyrosine kinase 2 (ERBB2) in patients with $\mathrm{PABC}$ has been demonstrated to lead to high proliferation of the tumors (7). Furthermore, C-X-C motif chemokine ligand 13 (CXCL13) and C-C motif chemokine ligand 20 (CCL2O) have also been demonstrated to be associated with the progression of PABC (8). Although potential biomarkers associated with PABC have been studied previously, it is far from enough for the diagnosis and treatment of PABC.

The breast consists of two major cell populations, stromal and epithelial cells, that communicate with each other through the extracellular matrix (9). Breast cancer manifests in the epithelium, but the involvement of stromal cells in tumorigenesis is also receiving attention (10). In the present study, gene expression profile data GSE31192 was downloaded to identify the differentially expressed genes (DEGs) between tumor associated stromal cells and normal stromal cells in PABC. Based on the obtained DEGs, Gene Ontology (GO) functional 
annotation, Kyoto Encyclopedia of Genes and Genomes (KEGG) pathway enrichment analysis and protein-protein interaction (PPI) network analysis were performed. Notably, in order to validate the findings of the present study, microarray data of GSE15852 were downloaded, and the DEGs between 43 breast tumors and their paired normal controls were identified. These DEGs were subsequently compared with those in GSE31192. The aim of the present study was to explore the DEGs in tumor-associated stroma of PABC using bioinformatics methods.

\section{Data and methods}

Affymetrix microarray data. The unstandardized gene expression profile microarray data GSE31192 was downloaded from the Gene Expression Omnibus (GEO; http://www.ncbi.nlm.nih. gov/geo/) (11) database of the National Center for Biotechnology Information, which was deposited by Harvell et al (9). The data platform was Affymetrix Human Genome U133 Plus 2.0 Array (Affymetrix, Inc.; Thermo Fisher Scientific, Inc., Waltham, MA, USA). The dataset included 33 samples that were obtained from women whose tumors arose while they were pregnant or within 1 year of delivery, and from age-matched controls who had never been pregnant. In the present study, 7 samples of tumor associated stromal cells and 4 samples of normal stromal cells in patients with PABC were selected to study the differences in gene expression.

Data preprocessing and DEGs analysis. All raw data were quantile normalized using the Robust Multiarray Averaging (RMA) method (12) in the Affy version 1.52.0 (http://www .bioconductor.org/packages/release/bioc/html/affy.html) (13) package in $\mathrm{R}$ language. In addition, preprocessing including RMA background correction and conversion of probe ID to gene symbol were performed. Eventually, the standardized gene expression matrix of samples was obtained. The DEGs between tumor associated stromal cells and normal stromal cells in PABC patients were analyzed with Limma version 3.30.11 (http://www.bioconductor .org/packages/release/bioc/html/limma.html) (14) package in $\mathrm{R}$ language. Student's t-tests were used to obtain the corresponding $\mathrm{P}$-value of the gene symbols. $\mathrm{P}<0.01$ and $\log _{2}$ fold change $(\mathrm{FC})>1$ were considered as the cut-off values.

GO and KEGG pathway enrichment analyses. GO (http://www.geneontology.org) database (15) is a tool for the functional unification of large-scale genomic data, which includes 3 categories: Biological process (BP), molecular function and cellular component. The KEGG (http://www.genome .ad.jp/kegg/) (16) database is used to classify correlating gene sets into their respective pathways. In the present study, the GO function and KEGG pathway enrichment analyses for the DEGs were performed using the Database for Annotation, Visualization and Integrated Discovery (DAVID version 6.8; https://david.ncifcrf.gov/) (17) online tool, which aimed to provide functional explanations of a large number of genes derived from genomic studies and proteins. With the enrichment thresholds of modified fisher exact $\mathrm{P}$-value $<0.05$ and the count number $>2$, the DEG enrichment results in the GO BP and KEGG pathway were obtained (16).
PPI network construction. Search Tool for the Retrieval of Interacting Genes (STRING version 10.0; http://string.embl .de/) (18) is a network resource and biological database of predicted and known PPIs. In this tool, the data of confidence scores were calculated for all protein interactions (19). In the present study, the DEGs were mapped into the STRING database to identify significant protein pairs with a combined score $>0.4$. Then, the PPI network was constructed based on the obtained PPI interaction pairs. Previous studies on biological networks have revealed that most PPI networks obeyed the scale-free attribution. Thus, the connectivity degree was analyzed using the network statistics to obtain the critical nodes, namely hub proteins (20).

Data validation based on GSE15852. In order to validate the reliability of the results, the expression profile microarray GSE15852 was downloaded from the GEO database based on the platform of GPL96 (HG-U133A) Affymetrix Human Genome U133A (Affymetrix Inc.; Thermo Fisher Scientific, Inc.). There were 86 samples in this dataset, including 43 breast tumors and their paired normal control.

The procedures of data preprocessing were the same as those detailed above. The DEGs between the 43 breast tumors and their paired normal control were then identified with the thresholds of $\mathrm{P}<0.01$ and $\log _{2} \mathrm{FC}>1$. The identified DEGs were subsequently compared with the DEGs identified in GSE31192.

\section{Results}

DEG analysis. In order to obtain DEGs between tumor-associated stromal cells and normal stromal cells in patients with PABC, the publicly available microarray dataset was downloaded from GEO and analyzed with the Limma package. With $\mathrm{P}<0.01$ and $\log _{2} \mathrm{FC}>1$, a total of 480 genes were identified, including 94 upregulated genes and 386 downregulated ones.

GO enrichment analysis of DEGs. To investigate the functional changes in the pathologic course of PABC, the identified DEGs were mapped to the GO database. Tables I and II list the top 10 GOBP enrichment results of upregulated and downregulated DEGs, respectively. The upregulated DEGs were primarily enriched in BP terms associated with the immune response (12 genes), regulation of cell proliferation (10 genes) and defense response (9 genes). For instance, interleukin 18 (IL18) and cluster of differentiation 274 (CD274) were primarily enriched in the immune response. The downregulated DEGs were enriched in cell surface receptor linked signal transduction (49 genes), cell adhesion (45 genes) and biological adhesion (45 genes).

KEGG pathway enrichment analysis of DEGs. To gain further insights into the changes of biological pathways in PABC, significant enrichment of the DEGs in was observed in multiple KEGG terms. The significant pathway enrichment results were listed in Tables III and IV. As revealed in Table III, only one significant pathway enriched by upregulated DEGs was obtained: The cytokine-cytokine receptor interaction pathway. In addition, 9 pathways of downregulated DEGs were obtained, including focal adhesion (21 genes), pathways 
Table I. GO biological process functional enrichment analysis results of upregulated genes (top 10).

\begin{tabular}{|c|c|c|c|c|}
\hline Category & Term & Description & Count & P-value \\
\hline $\mathrm{BP}$ & GO:0006955 & Immune response & 12 & $8.59 \times 10^{-5}$ \\
\hline $\mathrm{BP}$ & GO:0042127 & Regulation of cell proliferation & 10 & $4.15 \times 10^{-3}$ \\
\hline $\mathrm{BP}$ & GO:0006952 & Defense response & 9 & $3.21 \times 10^{-3}$ \\
\hline $\mathrm{BP}$ & GO:0007267 & Cell-cell signaling & 8 & $1.04 \times 10^{-2}$ \\
\hline BP & GO:0042981 & Regulation of apoptosis & 8 & $4.32 \times 10^{-2}$ \\
\hline $\mathrm{BP}$ & GO:0043067 & Regulation of programmed cell death & 8 & $4.52 \times 10^{-2}$ \\
\hline $\mathrm{BP}$ & GO:0010941 & Regulation of cell death & 8 & $4.60 \times 10^{-2}$ \\
\hline $\mathrm{BP}$ & GO:0051249 & Regulation of lymphocyte activation & 6 & $3.28 \times 10^{-4}$ \\
\hline $\mathrm{BP}$ & GO:0002694 & Regulation of leukocyte activation & 6 & $5.55 \times 10^{-4}$ \\
\hline $\mathrm{BP}$ & GO:0050865 & Regulation of cell activation & 6 & $7.05 \times 10^{-4}$ \\
\hline
\end{tabular}

Category represents GO functional classification. GO, Gene Ontology; BP, biological process.

Table II. Gene Ontology biological process P functional enrichment analysis results of downregulated genes (top 10).

\begin{tabular}{|c|c|c|c|c|}
\hline Category & Term & Description & Count & P-value \\
\hline $\mathrm{BP}$ & GO:0007166 & Cell surface receptor linked signal transduction & 49 & $1.14 \times 10^{-2}$ \\
\hline $\mathrm{BP}$ & GO:0007155 & Cell adhesion & 45 & $9.72 \times 10^{-13}$ \\
\hline $\mathrm{BP}$ & GO:0022610 & Biological adhesion & 45 & $1.01 \times 10^{-12}$ \\
\hline $\mathrm{BP}$ & GO:0007610 & Behavior & 29 & $5.78 \times 10^{-8}$ \\
\hline $\mathrm{BP}$ & GO:0010033 & Response to organic substance & 28 & $4.60 \times 10^{-4}$ \\
\hline $\mathrm{BP}$ & GO:0042127 & Regulation of cell proliferation & 28 & $1.70 \times 10^{-3}$ \\
\hline $\mathrm{BP}$ & GO:0042981 & Regulation of apoptosis & 27 & $4.56 \times 10^{-3}$ \\
\hline $\mathrm{BP}$ & GO:0043067 & Regulation of programmed cell death & 27 & $5.18 \times 10^{-3}$ \\
\hline $\mathrm{BP}$ & GO:0010941 & Regulation of cell death & 27 & $5.41 \times 10^{-3}$ \\
\hline $\mathrm{BP}$ & GO:0010604 & Positive regulation of macromolecule metabolic process & 27 & $1.01 \times 10^{-2}$ \\
\hline
\end{tabular}

Category represents GO functional classification. GO, Gene Ontology; BP, biological process.

Table III. KEGG pathway enrichment analysis results of upregulated genes.

\begin{tabular}{lcccc}
\hline Category & Term & Description & Count & P-value \\
\hline KEGG & hsa04060 & $\begin{array}{l}\text { Cytokine-cytokine } \\
\text { receptor interaction }\end{array}$ & 6 & $7.94 \times 10^{-3}$ \\
\hline
\end{tabular}

Category represents KEGG pathway. KEGG, Kyoto Encyclopedia of Genes and Genomes.

in cancer (17 genes), ECM-receptor interaction (13 genes), and vascular smooth muscle contraction (7 genes).

PPI network construction. In order to obtain novel insights into protein function, the PPI network was constructed. Using STRING (combined score $>0.4$ ), a total of 286 nodes and 811 protein interaction pairs were obtained (Fig. 1). The nodes with degree $\geq 10$ in the PPI network were listed in Table V, including jun proto-oncogene $(J U N$; degree $=61)$, FBJ murine osteosarcoma viral oncogene homolog $(F O S$; degree $=50)$, epidermal growth factor receptor $(E G F R$; degree=48), V-myc avian myelocytomatosis viral oncogene homolog (MYC; degree $=32$ ), and $\alpha$-smooth muscle actin (ACTA2; degree $=21)$.

Data validation based on GSE15852. Following data validation of the DEGs based on GSE15852 microarray data, the hub genes obtained in GSE31192, including JUN, FOS, EGFR, $M Y C$ and ACTA2 were revealed to have the same expression levels as in GSE15852. In addition, IL18 (enriched in BP terms associated with the immune response) was also upregulated in GSE15852. The identified DEGs in GSE15852 can be obtained from http://pan.baidu.com/share/link? shareid=9888 $37841 \& u k=3125911049$.

\section{Discussion}

PABC is a malignant tumor that poses a serious threat to women's health (21). Therefore, exploration of the pathogenesis of PABC is urgent. In the present study, based on the predefined thresholds of the software and online tools used, a 
Table IV. KEGG pathway enrichment analysis results of downregulated genes.

\begin{tabular}{|c|c|c|c|c|}
\hline Category & Term & Description & Count & P-value \\
\hline KEGG & hsa04510 & Focal adhesion & 21 & $2.29 \times 10^{-8}$ \\
\hline KEGG & hsa05200 & Pathways in cancer & 17 & $3.45 \times 10^{-3}$ \\
\hline KEGG & hsa04512 & ECM-receptor interaction & 13 & $3.84 \times 10^{-7}$ \\
\hline KEGG & hsa05210 & Colorectal cancer & 8 & $3.14 \times 10^{-3}$ \\
\hline KEGG & hsa05215 & Prostate cancer & 8 & $4.34 \times 10^{-3}$ \\
\hline KEGG & hsa04360 & Axon guidance & 8 & $2.96 \times 10^{-2}$ \\
\hline KEGG & hsa04012 & ErbB signaling pathway & 7 & $1.51 \times 10^{-2}$ \\
\hline KEGG & hsa04270 & Vascular smooth muscle contraction & 7 & $4.50 \times 10^{-2}$ \\
\hline KEGG & hsa05222 & Small cell lung cancer & 6 & $4.47 \times 10^{-2}$ \\
\hline
\end{tabular}

KEGG, Kyoto Encyclopedia of Genes and Genomes.

Table V. Nodes with higher connectivity degree in the PPI network of differentially expressed genes (degree $\geq 10)$.

\begin{tabular}{|c|c|c|c|c|c|c|c|}
\hline Node & Degree & Node & Degree & Node & Degree & Node & Degree \\
\hline$J U N$ & 61 & $L P L$ & 17 & EPHA2 & 14 & $M M P 3$ & 12 \\
\hline FOS & 50 & NOTCH4 & 16 & PENK & 13 & OXTR & 12 \\
\hline$E G F R$ & 48 & $A B L 1$ & 16 & $T N C$ & 13 & $C D 36$ & 12 \\
\hline$M Y C$ & 32 & $A C T G 2$ & 16 & $E G R 2$ & 13 & $P D E 7 B$ & 11 \\
\hline EGRI & 27 & $N G F R$ & 16 & $B D K R B 2$ & 13 & $L A M B 2$ & 11 \\
\hline$I G F 1$ & 23 & COLAA2 & 16 & ITGA7 & 13 & $N E S$ & 11 \\
\hline PDGFRB & 22 & PLCBI & 16 & $F O S B$ & 13 & $H S D 17 B 6$ & 10 \\
\hline ACTA2 & 21 & ATF3 & 15 & TCF7L2 & 13 & RASL1OA & 10 \\
\hline LPAR1 & 19 & GNRH1 & 15 & DUSPI & 13 & $M M E$ & 10 \\
\hline$V W F$ & 19 & ITGA5 & 14 & LPAR5 & 13 & EGR3 & 10 \\
\hline COL18A1 & 18 & NR4Al & 14 & $E D N R B$ & 12 & & \\
\hline PTGS2 & 17 & $T A C 1$ & 14 & NR4A2 & 12 & & \\
\hline
\end{tabular}

Node represents specific gene (protein) name (symbol), Degree represents the gene connectivity degree in the PPI network. PPI, protein-protein interaction.

total of 480 DEGs were identified between tumor stromal cells and normal stromal cells in patients with PABC, including JUN, FOS, MYC, ACTA2, IL18 and CD274. These genes were primarily enriched in pathways in cancer, and vascular smooth muscle contraction, and in GO BP terms were associated with cell surface receptor linked signal transduction and the immune response. Furthermore, the DEGs of JUN, FOS, MYC and $A C T A 2$ were hub genes with higher degrees in the PPI network. Notably, these DEGs, with the exception of CD274, were validated by the dataset of GSE15852.

By analyzing the DEGs in the PPI network, JUN, FOS, $M Y C$ and ACTA2 were revealed to be hub genes with higher degrees. JUN and FOS are proto-oncogenes which are involved in multiple tumors. $J U N$ is the component of transcription factor activator protein 1 (AP-1), which primarily forms heterodimers with $F O S$, and was the first oncogenic transcription factor to be discovered (22). AP-1 participates in multiple cellular processes, including cell proliferation, transformation and death, and is also involved in tumorigenesis, proliferation and metastasis. $J U N$ is activated by c-jun
N-terminal kinases (JNKs) through c-jun N-terminal phosphorylation of the JNK pathway (23). The binding of JUN and FOS to a high-affinity AP-1 binding site on DNA induces gene transcription and promotes tumorigenesis (24). Previous studies have demonstrated that human chorionic gonadotropin in the breast cancer Michigan Cancer Foundation-7 cell line exerts anti-proliferative and anti-invasive effects by downregulating nuclear factor- $\kappa \mathrm{B}$ and AP-1 transcription factors, so as to induce a protective effect in pregnant women (25). Notably, a previous study has also reported that deregulation of $J U N$ expression results in the metastasis of breast cancer (26). Therefore, deregulation of JUN and FOS maybe involved in the tumor-associated stroma of PABC.

$M Y C$ is a regulator gene coding for a transcription factor that is involved in cell cycle progression, apoptosis and cellular transformation (27). It is activated by a variety of mitogenic signals, including EGF, wingless and INT-1 via the mitogen-activated protein kinases/extracellular signal-regulated kinases pathway (28). Imbalanced expression of $M Y C$ promotes cell transformation from $\mathrm{G} 1$ to $\mathrm{S}$ phase, thereby 


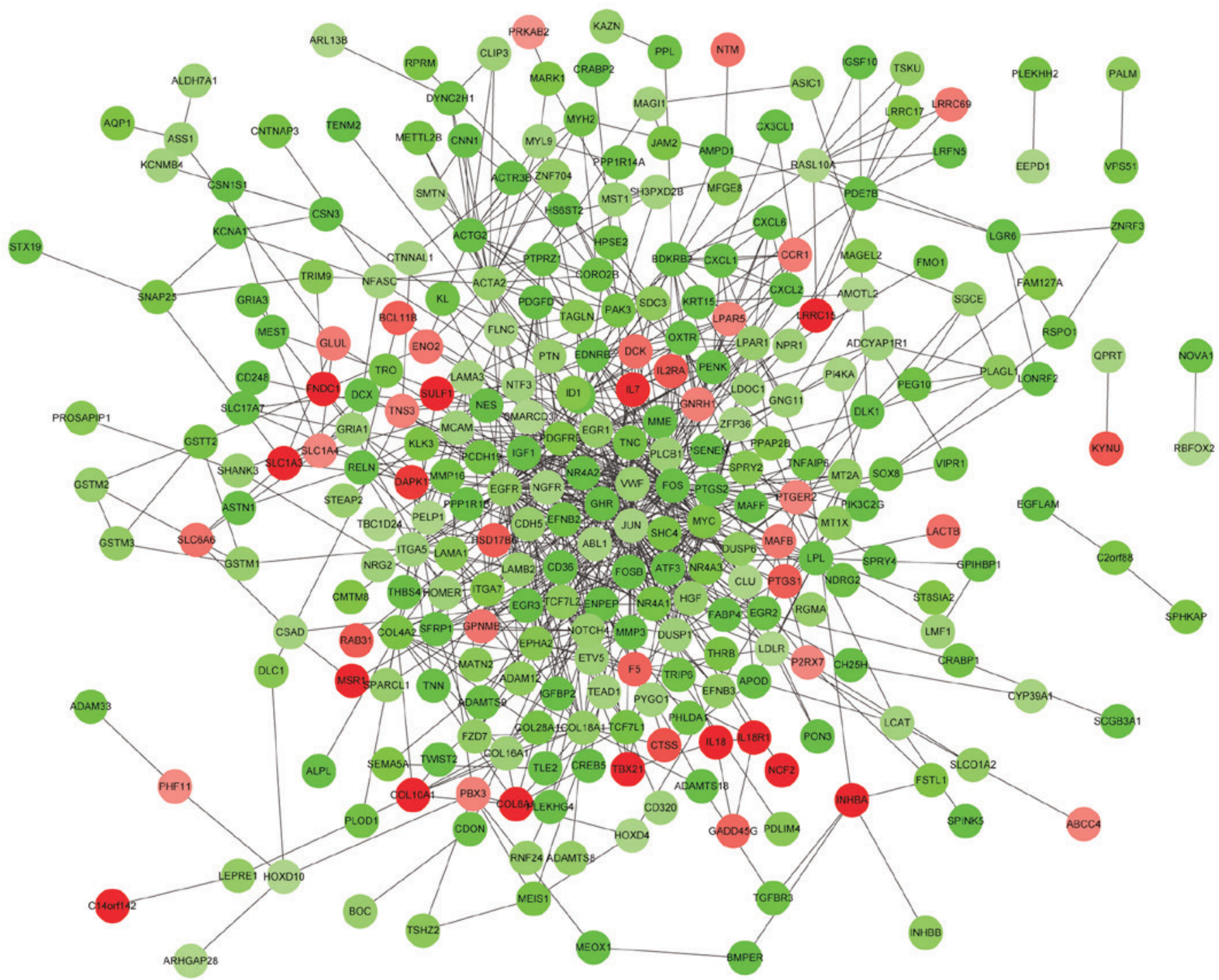

Figure 1. Protein-protein interaction network for the differentially expressed genes in tumor-associated stromal cells of pregnancy-associated breast cancer. There were 286 nodes and 811 edges (protein interaction pairs) in the network. The red nodes represent upregulated genes and the green nodes represent downregulated genes. The color depth represents the strength of significance. The deeper color represents a smaller P-value compared with the normal cells.

leading to cell proliferation and the formation of cancer (29). Notably, malfunctions in MYC have been observed in several types of cancer, including breast, uterine, gastric, pancreatic and colorectal cancer (30). MYC is thus considered to be a promising target for anti-cancer drugs. Taken together, it was hypothesized that $M Y C$ may act as a potential biomarker in tumor-associated stroma of PABC.

Another hub gene, ACTA2, encodes a protein which belongs to the highly conserved actin family. It is known to contribute to cell-generated mechanical tension and be involved in cell motility, structure and integrity (31). In the present study, ACTA2 was demonstrated to be enriched in the pathway of vascular smooth muscle contraction. Lambrechts et al (32) revealed that ACTA2 is primarily expressed in the smooth muscle cells and activated cancer-associated fibroblasts. Tumor cells use actin bundles to allow them to break away from a primary tumor and invade the surrounding tissue (33). ACTA2 has been demonstrated to be involved in lung adenocarcinoma metastasis (31). Therefore, the deregulation of ACTA2 in tumor stroma may affect the progression of PABC.
Furthermore, previous studies have demonstrated that the expression changes of genes associated with the immune response contribute to tumor aggressiveness (34). The analysis data of Ma et al (35) revealed that the upregulated genes associated with the immune response are exhibited in the stroma of high-grade PABC. In the present study, the immune response was a significant BP term that was enriched by $I L 18$ and $C D 274$. IL18 is a tumor inhibiting factor, dysregulation of which has been observed in the progression of tumors. Studies have reported that IL18 effectively inhibits the growth of hepatoma cells by inhibiting angiogenesis and participating in apoptotic signal transduction (36). An analysis of tumor specimens with renal cell carcinoma has revealed that high expression of CD274 increases tumor aggressiveness and risk of death (37). Thus, IL18 and CD274 may affect the progression of PABC through the pathways associated with immune response.

However, the present study has limitations. In the process of data analysis, only a few samples were used. In addition, no microarray experiments were performed, except for bioinformatics analysis of two gene expression profile data from the 
GEO database. Therefore, further experimental studies with larger sample size are required to confirm the observations of the present study.

In conclusion, there were significant differences in gene expression between tumor-associated stromal cells and normal stromal cells in PABC, confirmed by analyzing the gene expression profiles with bioinformatics. The DEGs of JUN, FOS, MYC, ACTA2, IL18 and CD274, and BP terms associated with the immune response may be involved in the development of tumor stroma in PABC.

\section{Acknowledgements}

The present study was supported by the Key Program of Science and Technology Development Fund of Nanjing Medical University (grant no. 2013NJMU146).

\section{References}

1. Sathian B, Nagaraja SB, Banerjee I, Sreedharan J, De A, Roy B, Rajesh E, Senthilkumaran S, Hussain SA and Menezes RG: Awareness of breast cancer warning signs and screening methods among female residents of Pokhara valley, Nepal. Asian Pac J Cancer Prev 15: 4723-4726, 2014.

2. Rovera F, Frattini F, Coglitore A, Marelli M, Rausei S, Dionigi G, Boni L and Dionigi R: Breast cancer in pregnancy. Breast J 16 (Suppl 1): S22-S25, 2010.

3. Abenhaim HA, Azoulay L, Holcroft CA, Bure LA, Assayag J and Benjamin A: Incidence, risk factors, and obstetrical outcomes of women with breast cancer in pregnancy. Breast J 18: 564-568, 2012.

4. Antoniou A, Pharoah P, Narod S, Risch HA, Eyfjord JE, Hopper JL, Loman N, Olsson H, Johannsson O, Borg A, et al: Average risks of breast and ovarian cancer associated with BRCA1 or BRCA2 mutations detected in case series unselected for family history: A combined analysis of 22 studies. Am J Hum Genet 72: 1117-1130, 2003.

5. Cullinane CA, Lubinski J, Neuhausen SL, Ghadirian P, Lynch HT, Isaacs C, Weber B, Moller P, Offit K, Kim-Sing C, et al: Effect of pregnancy as a risk factor for breast cancer in BRCA1/BRCA2 mutation carriers. Int J Cancer 117: 988-991, 2005.

6. Kotsopoulos J, Lubinski J, Lynch HT, Klijn J, Ghadirian P, Neuhausen SL, Kim-Sing C, Foulkes WD, Moller P, Isaacs C, et al: Age at first birth and the risk of breast cancer in BRCA 1 and BRCA2 mutation carriers. Breast Cancer Res Treat 105: 221-228, 2007.

7. Gao L, Ding H, Li L and Hu H: A clinicopathological study of pregnancy-associated breast cancer and breast cancer with pregnancy-like change. Chin J Clin Exper Pathol 20: 29-34, 2003 (In Chinese).

8. Douglas MR, Morrison KE, Salmon M and Buckley CD: Why does inflammation persist: A dominant role for the stromal microenvironment? Expert Rev Mol Med 4: 1-18, 2002.

9. Harvell DM, Kim J, O'Brien J, Tan AC, Borges VF, Schedin P, Jacobsen BM and Horwitz KB: Genomic signatures of pregnancy-associated breast cancer epithelia and stroma and their regulation by estrogens and progesterone. Horm Cancer 4: 140-153, 2013.

10. Wiseman BS and Werb Z: Stromal effects on mammary gland development and breast cancer. Science 296: 1046-1049, 2002

11. Edgar R, Domrachev M and Lash AE: Gene expression omnibus: NCBI gene expression and hybridization array data repository. Nucleic Acids Res 30: 207-210, 2002.

12. Irizarry RA, Hobbs B, Collin F, Beazer-Barclay YD, Antonellis KJ, Scherf U and Speed TP: Exploration, normalization, and summaries of high density oligonucleotide array probe level data. Biostatistics 4: 249-264, 2003.

13. Gautier L, Cope L, Bolstad BM and Irizarry RA: Affy-analysis of Affymetrix GeneChip data at the probe level. Bioinformatics 20: 307-315, 2004

14. Ritchie ME, Phipson B, Wu D, Hu Y, Law CW, Shi W and Smyth GK: limma powers differential expression analyses for RNA-sequencing and microarray studies. Nucleic Acids Res 43: e47, 2015.
15. Ashburner M, Ball CA, Blake JA, Botstein D, Butler $\mathrm{H}$, Cherry JM, Davis AP, Dolinski K, Dwight SS, Eppig JT, et al: Gene ontology: Tool for the unification of biology. The Gene ontology consortium. Nat Genet 25: 25-29, 2000.

16. Kanehisa M and Goto S: KEGG: Kyoto encyclopedia of genes and genomes. Nucleic Acids Res 28: 27-30, 2000.

17. Huang DW, Sherman BT, Tan Q, Collins JR, Alvord WG, Roayaei J, Stephens R, Baseler MW, Lane HC and Lempicki RA: The DAVID gene functional classification tool: A novel biological module-centric algorithm to functionally analyze large gene lists. Genome Biol 8: R183, 2007.

18. Von Mering C, Huynen M, Jaeggi D, Schmidt S, Bork P and Snel B: STRING: A database of predicted functional associations between proteins. Nucleic Acids Res 31: 258-261, 2003.

19. Jensen LJ, Kuhn M, Stark M, Chaffron S, Creevey C, Muller J, Doerks T, Julien P, Roth A, Simonovic M, et al: STRING 8-a global view on proteins and their functional interactions in 630 organisms. Nucleic Acids Res 37 (Database issue): D412-D416, 2009.

20. He $X$ and Zhang J: Why do hubs tend to be essential in protein networks? PLoS Genet 2: e88, 2006.

21. Andersson TM, Johansson AL, Hsieh CC, Cnattingius S and Lambe M: Increasing incidence of pregnancy-associated breast cancer in Sweden. Obstet Gynecol 114: 568-572, 2009.

22. Vogt PK: Fortuitous convergences: The beginnings of JUN. Nat Rev Cancer 2: 465-469, 2002.

23. Behrens A, Sibilia M and Wagner EF: Amino-terminal phosphorylation of c-Jun regulates stress-induced apoptosis and cellular proliferation. Nat Genet 21: 326-329, 1999.

24. Rauscher FD III, Voulalas P, Franza B Jr and Curran T: Fos and Jun bind cooperatively to the AP-1 site: Reconstitution in vitro. Genes Dev 2: 1687-1699, 1988.

25. Rao ChV, Li X, Manna SK, Lei ZM and Aggarwal BB: Human chorionic gonadotropin decreases proliferation and invasion of breast cancer MCF-7 cells by inhibiting NF-kappaB and AP-1 activation. J Biol Chem 279: 25503-25510, 2004.

26. Zhang Y, Pu X, Shi M, Chen L, Song Y, Qian L, Yuan G, Zhang $\mathrm{H}, \mathrm{Yu} \mathrm{M}, \mathrm{Hu} \mathrm{M}$, et al: Critical role of c-Jun overexpression in liver metastasis of human breast cancer xenograft model. BMC Cancer 7: 145, 2007.

27. Facchini LM and Penn LZ: The molecular role of Myc in growth and transformation: Recent discoveries lead to new insights. FASEB J 12: 633-651, 1998.

28. Hayashi J, Aoki H, Kajino K, Moriyama M, Arakawa Y and Hino O: Hepatitis $C$ virus core protein activates the MAPK/ERK cascade synergistically with tumor promoter TPA, but not with epidermal growth factor or transforming growth factor alpha. Hepatology 32: 958-961, 2000.

29. Santoni-Rugiu E, Falck J, Mailand N, Bartek J and Lukas J: Involvement of Myc activity in a G1/S-promoting mechanism parallel to the pRb/E2F pathway. Mol Cell Biol 20: 3497-3509, 2000.

30. Chen Y, McGee J, Chen X, Doman TN, Gong X, Zhang Y, Hamm N, Ma X, Higgs RE, Bhagwat SV, et al: Identification of druggable cancer driver genes amplified across TCGA datasets. PloS One 9: e98293, 2014

31. Lee HW, Park YM, Lee SJ, Cho HJ, Kim DH, Lee JI, Kang MS, Seol HJ, Shim YM, Nam DH, et al: Alpha-smooth muscle actin (ACTA2) is required for metastatic potential of human lung adenocarcinoma. Clin Cancer Res 19: 5879-5889, 2013.

32. Lambrechts A, Van Troys M and Ampe C: The actin cytoskeleton in normal and pathological cell motility. Int J Biochem Cell Biol 36: 1890-1909, 2004.

33. Fritz G and Kaina B: Rho GTPases: Promising cellular targets for novel anticancer drugs. Curr Cancer Drug Targets 6: 1-14, 2006.

34. Asztalos S, Gann PH, Hayes MK, Nonn L, Beam CA, Dai Y, Wiley EL and Tonetti DA: Gene expression patterns in the human breast after pregnancy. Cancer Prev Res (Phila) 3: 301-311, 2010.

35. Ma XJ, Dahiya S, Richardson E, Erlander M and Sgroi DC: Gene expression profiling of the tumor microenvironment during breast cancer progression. Breast Cancer Res 11: R7, 2009.

36. Wenfeng Y: Interleukin-18 anti-hepatocellular carcinoma in nude mice transplanted mechanism. Soochow University, 2009 (In Chinese).

37. Thompson RH, Gillett MD, Cheville JC, Lohse CM, Dong H, Webster WS, Krejci KG, Lobo JR, Sengupta S, Chen L, et al: Costimulatory B7-H1 in renal cell carcinoma patients: Indicator of tumor aggressiveness and potential therapeutic target. Proc Natl Acad Sci USA 101: 17174-17179, 2004. 